\title{
Estágios de mudança de comportamento e percepção positiva do ambiente para atividade física em usuários de parque urbano
}

\author{
E.L. Petroski, D.A.S. Silva, R.S. Reis, A. Pelegrini
}

Os objetivos do estudo foram: (a) identificar os estágios de mudança de comportamento (EMC) para atividade física (AF) em usuários de parques urbanos, (b) verificar a associação entre os indicadores sócio-demográficos e econômicos com a prática de $\mathrm{AF}$ em usuários de parques urbanos, (c) analisar os indicadores ambientais percebidos positivamente para a prática de AF. Participaram do estudo 218 usuários de um parque urbano que preencheram um questionário com informações sócio-demográficas, EMC e percepção do ambiente existente. Proporção mais elevada de usuários foi encontrada nos estágios manutenção $(56,6 \%)$ e ação $(21,1 \%)$. A maioria $(78,7 \%)$ dos usuários possui comportamento ativo (manutenção+ação) à prática de $A F$, sendo mais prevalente nos indivíduos de meiaidade $(p<0,05)$. A beleza e localização geográfica do parque, os fatores tecnológicos e arquitetônicos, alguns políticos normativos (serviços públicos à disposição dos usuários) e de valores e atitudes foram percebidos positivamente para AF independente de os usuários praticarem ou não AF regularmente. $O$ estudo sugere que a maior parte dos usuários do parque público apresenta comportamento ativo à prática de $A F$, sobretudo, os de meia-idade. Ademais, independente do EMC em relação à $A F$, a percepção dos indicadores ambientais é positiva entre os usuários sedentários e ativos fisicamente.

Palavras-chave: atividade motora, parques nacionais, atividades de lazer, comportamento

Submetido: 18.04.2009| Aceite: 09.06.2009

Edio Luiz Petroski. Universidade Federal de Santa Catarina/Centro de Desportos/Núcleo de Pesquisa em Cineantropometria e Desempenho Humano. Florianópolis, SC, Brasil.

Rodrigo Siqueira Reis. Universidade Federal do Paraná/Programa de Pós-Graduação em Educação Física. Curitiba, PR, Brasil. Pontifícia Universidade Católica de Curitiba.

Diego Augusto Santos Silva e Andreia Pelegrini. Universidade Federal de Santa Catarina/Centro de Desportos/Núcleo de Pesquisa em Cineantropometria e Desempenho Humano. Florianópolis, SC, Brasil. Bolsistas Capes.

Endereço para correspondência: Edio Luiz Petroski, Universidade Federal de Santa Catarina Centros de Desportos, Núcleo de Pesquisa em Cineantropometria e Desempeno Humano, Campus Universitário - Trindade - Caixa Postal 476, CEP 88040-900 - Florianópolis, SC, Brasil. E-mail: petroski@cds.ufsc.br 


\section{Stages of behavioral change and positive perception of the environment towards physical activity among urban park users}

The objectives of this study were to identify the stages of behavioral change (SBC) towards physical activity (PA) among users of urban parks, to determine the association between socio-demographic and economic variables of users of urban parks in relation to physical activity (PA), and to analyze environmental indicators of the park perceived positively for PA. A total of 218 users of an urban park, who filled out a questionnaire regarding socio-demographic data, SBC and perception of the existing environment, participated in the study. The most prevalent SBC were maintenance $(56.6 \%)$ and action $(21.1 \%)$. Most park users $(78.7 \%)$ presented an active behavior (maintenance + action) towards PA, with this behavior being prevalent among middle-aged individuals $(p<0.05)$. The beauty and geographic location of the park, technological and architectonic factors, some normative policies (public services to the users' disposition), and values and attitudes were perceived positively for PA, regardless of whether the users regularly performed PA or not. The study suggests that most public park users present an active behavior towards PA, especially middle-aged individuals. In addition, the perception of environmental indicators is positive among physically inactive and active park users, irrespective of SBC.

Key words: motor activity, national parks, leisure activities, behavior

A identificação de como as pessoas percebem o ambiente que frequentam é utilizada como um meio inicial para avaliação em promoção da saúde (Cervantes, Espejel, Arellano, \& Delhumeau, 2008; Marchisio, Sarasino, Manzoli, Di Stanislao, $\&$ Panella, 2007). Pesquisas desta natureza, realizadas com usuários de centros de saúde (Cunningham et al., 2005), bairros (Righi, Aggazzotti, Fantuzzi, Ciccarese, \& Predieri, 2002), ambientes de trabalho (Marchisio et al., 2007) e lazer (Cervantes et al., 2008) foram capazes de sugerir modificações em nível organizacional e estrutural para propiciar comportamentos mais saudáveis.

Os parques urbanos são considerados ambientes adequados para a prática de lazer e de atividade física (AF). Por isso, a construção e revitalização destes locais têm recebido especial atenção por órgãos internacionais como forma de promover e incentivar um estilo de vida ativo (Librett, Henderson, Godbey, \& Morrow, 2007). Alguns determinantes ambientais como a acessibilidade, disponibilidade, segurança, tráfego urbano, condições climáticas, atmosféricas, suporte social, crenças e atitudes são percebidos como influenciadores da AF em parques (Bedimo-Rung, Mowen, \& Cohen, 2005; Cohen, Meister, \& Zapien, 2004). 
O indivíduo é o foco da prática de promoção da saúde, sendo que teorias de nível individual centram quase que exclusivamente em fatores intrapessoais, as quais são utilizadas para entender o processo e a adoção de um comportamento mais saudável (Green, et al., 1994). Dentre as teorias, destacam-se os estágios de mudança de comportamento (EMC), conhecido também como modelo Transteorético. A premissa básica deste, que é um processo e não um acontecimento, é a mudança de comportamento, haja vista que cada pessoa apresenta níveis diferentes de motivação, ou disposição para mudar seus hábitos (Prochaska \& DiClemente, 1982).

No modelo Transteorético são identificados cinco estágios (pré-contemplação, contemplação, preparação, ação e manutenção), sendo que tais estágios seguem uma ordem do "menos saudável" para o "mais saudável". Além disso, a classificação dos EMC permite distinguir os indivíduos que estão dispostos a fazer mudanças no seu comportamento daqueles que não pretendem.

Os EMC relacionados à AF foram aplicados pela primeira vez em 1992 (Marcus, Rossi, Selby, Niaura, \& Abrams, 1992), e hoje também é considerado um instrumento relevante para distinguir o padrão da prática de AF das pessoas (Nigg, 2005).

Alguns estudos, que utilizaram os $E M C$ relacionados à $A F$, foram realizados em universidades (Petroski \& Martins, 2008), comunidades (Marcus et al., 1992) e serviços de saúde (Norris, Grothaus, Buchner, \& Pratt, 2000), os quais serviram para subsidiar políticas de promoção da AF. Todavia, não se tem conhecimento da utilização deste modelo com usuários de parques urbanos. Portanto, a identificação da percepção do ambiente pelos usuários, bem como de suas características sócio-demográficas e econômicas, poderiam auxiliar na compreensão dos fatores que possivelmente influenciam na ocupação de parques urbanos enquanto espaço de lazer.

Assim, o presente estudo tem como objetivos:

(a) identificar os EMC para AF em usuários de parques urbanos,

(b) verificar a associação entre os indicadores sócio-demográficos (sexo, faixa etária, estado civil) e nível econômico com a prática de AF em usuários de parques urbanos,

(c) analisar os indicadores ambientais (geográficos, tecnológicos e arquitetônicos, políticos normativos, de valores e atitudes) percebidos positivamente para a prática de AF em usuários ativos e sedentários. 


\section{Metodologia}

Este estudo, com delineamento transversal, caracterizado como descritivo, foi aprovado pelo Comitê de Ética em Pesquisa da Pontifícia Universidade Católica do Paraná. A população do estudo foi formada de usuários adultos ( $\geq 18$ anos de idade) do parque Jardim Botânico da cidade de Curitiba, Paraná, Brasil. Este parque localiza-se no bairro Jardim Botânico que conta com uma população estimada de 7.000 pessoas.

\section{Amostra}

O processo para seleção da amostra desenvolveu-se em duas etapas: no primeiro momento, foi realizada uma entrevista estruturada com os frequentadores do parque. Nesta etapa, 843 pessoas foram entrevistadas durante 14 dias consecutivos, acerca de informações demográficas (sexo, idade, estado civil, nível de escolaridade e endereço), e se tinham interesse em participar no segundo momento da pesquisa em curso. Esta estratégia foi utilizada para que a amostra pudesse representar de forma mais abrangente a população usuária.

Na segunda etapa da amostragem, do tipo intencional, incluiu-se 721 sujeitos que aceitaram participar no segundo momento do estudo, que consistia em enviar, via correio, um instrumento auto-administrado e o Termo de Consentimento Livre e Esclarecido (TCLE), para ser preenchido, assinado, e devolvido para os pesquisadores.

Dos questionários enviados $(n=721)$, retornaram $256(35,5 \%)$ e, após a primeira verificação destes, foram excluídos 38 por terem respostas incompletas ou ausência de respostas nas variáveis consideradas no presente estudo.

Assim, com uma amostra de 218 sujeitos (30,2\%) procedeu-se à análise dos dados, uma vez que com este tamanho foram satisfeitos os critérios sugeridos na literatura, a qual sugere que estudos de levantamento por correio, um retorno próximo dos 30\% é considerado satisfatório (Mitra \& Lankfort, 1999).

\section{Instrumentos}

O instrumento auto-administrado enviado pelo correio era composto por quatro partes: 1) informações demográficas (sexo, estado civil, idade, escolaridade completa); 2) nível econômico (NE) (ABEP, 2003); 3) identificação do EMC (Marcus, et al., 1992) em relação à $A F$; 4 ) escala de percepção do ambiente existente (Reis, Nascimento, \& Petroski, 2002). 
O questionário utilizado para verificar o NE $(A B E P, 2003)$ utiliza um sistema de pontos que, somados, servem para dividir a população brasileira conforme sua capacidade de compra. As classes dos critérios adotados pela ABEP (2003) são cinco: $A, B, C, D, E$ por ordem decrescente de poder de compra, sendo as classes $A$ e $B$, divididas em $A_{1}, A_{2}$ e $B_{1}, B_{2}$ respectivamente. No presente estudo, o NE foi dividido em três classes: $A$ (alta), $B$ (média), $C+D+E$ (baixa).

Os EMC relacionados à atividade física foram verificados por meio de questionário (Marcus et al., 1992), os quais classificam os sujeitos em um dos cinco estágios: (1) Pré-Contemplação (o sujeito não pretende modificar seu comportamento num futuro próximo), (2) Contemplação (existe a intenção de mudar, mas não imediatamente), (3) Preparação (existe a intenção de mudar nos próximos seis meses), (4) Ação (mudanças recentes no comportamento), (5) Manutenção (as mudanças no comportamento já são mantidas por mais de seis meses).

No presente estudo, foi considerado comportamento "sedentário" os usuários que indicaram os estágios 1, 2 e 3, e "ativo" aqueles que indicaram os estágios 4 e 5 . Evidências de eficácia, de sensibilidade e especificidade quanto ao uso desta classificação podem ser obtidas na literatura (Nigg, 2005).

Para verificar a percepção dos usuários em relação ao ambiente existente, foi utilizada uma escala composta por 16 itens (Reis, Nascimento, \& Petroski, 2002). Esta escala aborda dimensões de ordem física e sócio-cultural do ambiente, abrangendo cinco fatores: 1) geográficos, que estão ligados a aspectos climáticos e de localização do parque, 2) tecnológico e arquitetônico, que são relacionados ao ambiente construído e a disponibilidade do mesmo, 3) político normativo, que possuem ligação com serviços públicos à disposição dos usuários, 4) valores e atitudes, que são relacionados com o suporte social.

Os itens do instrumento referem-se aos indicadores do ambiente percebidos como inibidores ou estimuladores da realização de AF no parque. As respostas são apresentadas em uma escala ordinal de 1 a 4 , em que o pólo negativo é composto pelas categorias "inibe bastante" (1) e "inibe" (2) e, o pólo positivo compreendido pelas categorias "estimula" (3) e "estimula bastante" (4).

Como o presente estudo visou identificar a percepção positiva do ambiente existente no parque, foram agrupadas e analisadas somente as categorias 3 e 4, pois estão associadas como fatores positivos à prática de AF. 


\section{Procedimentos}

Para análise dos dados, foi utilizada a estatística descritiva para caracterizar a amostra quanto aos EMC e a percepção dos indicadores ambientais. Para verificar associação entre as variáveis econômicas e sócio-demográficas e o comportamento em relação à $A F$, empregou-se o teste qui-quadrado. Para identificar diferenças entre duas proporções utilizou-se o teste de comparação entre proporções.

Em todas as análises adotou-se um nível de significância de 5\%.

\section{Resultados}

A figura 1 descreve a amostra quanto aos EMC em relação à AF. A maioria dos usuários do parque Jardim Botânico encontrava-se no estágio de manutenção $(54,6 \%)$ e a minoria $(0,5 \%)$ no de pré-contemplação. Dos usuários investigados, $24,3 \%$ apresentavam comportamento "sedentário" para AF e 75,7\% apresentavam um comportamento "ativo" para a prática de atividade física.

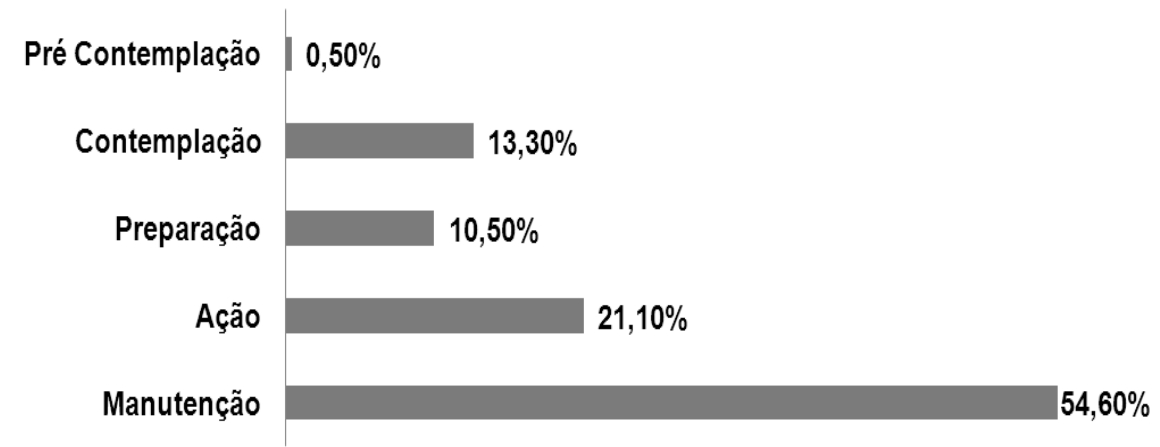

Figura 1. Proporção dos usuários do parque Jardim Botânico quanto aos EMC relacionados à AF. Curitiba, PR

$\mathrm{Na}$ tabela 1, observa-se a associação dos indicadores sócio-demográficos, econômicos dos usuários de parques urbanos em relação ao padrão de atividade AF.

Somente a faixa etária esteve associada com os $E M C$ em relação à $A F(p<0,05)$. Estes resultados indicam que o comportamento sedentário foi maior nos indivíduos com idade inferior a 40 anos (39\%). Já no comportamento ativo para a prática de $A F$, proporção mais elevada foi encontrada nos usuários de 40-59 anos de idade $(87 \%)$, seguidos daqueles com idade igual ou superior a 60 anos $(75 \%)$. 
Tabela 1. Associação entre os indicadores sócio-demográficos e econômicos de usuários de parques urbanos em relação ao padrão de atividade $\mathrm{AF}$

\begin{tabular}{|c|c|c|c|c|c|}
\hline \multirow{3}{*}{ Variáveis } & \multicolumn{4}{|c|}{ Comportamento em relação AF } & \multirow{3}{*}{$p$} \\
\hline & \multicolumn{2}{|c|}{ Sedentário } & \multicolumn{2}{|c|}{ Ativo } & \\
\hline & $n$ & $\%$ & $n$ & $\%$ & \\
\hline Sexo & & & & & 0,311 \\
\hline Masculino & 25 & 21,6 & 91 & 78,4 & \\
\hline Feminino & 28 & 27,5 & 74 & 72,5 & \\
\hline Faixa etária & & & & & $<0,001^{*}$ \\
\hline$<40$ & 32 & 39,0 & 50 & 61,0 & \\
\hline $40-59$ & 14 & 13,0 & 94 & 87,0 & \\
\hline$\geq 60$ & 7 & 25,0 & 21 & 75,0 & \\
\hline NE & & & & & 0,184 \\
\hline Alto & 14 & 17,5 & 66 & 82,5 & \\
\hline Médio & 29 & 27,4 & 77 & 72,6 & \\
\hline Baixo & 10 & 31,2 & 22 & 68,8 & \\
\hline Escolaridade & & & & & 0,500 \\
\hline Fundamental & 7 & 26,9 & 19 & 73,1 & \\
\hline Médio & 21 & 28,4 & 53 & 71,6 & \\
\hline Superior & 25 & 21,2 & 93 & 78,8 & \\
\hline Estado Civil & & & & & 0,138 \\
\hline Casado & 27 & 20,8 & 103 & 79,2 & \\
\hline Não Casado & 26 & 29,5 & 62 & 70,5 & \\
\hline
\end{tabular}

* $p<0,05$ - teste Qui-quadrado.

A tabela 2 descreve a proporção de indivíduos ativos e sedentários que perceberam positivamente os indicadores do ambiente para a prática de AF no parque. Os resultados demonstram que usuários com comportamento sedentário e ativo percebem de forma similar os indicadores ambientais para a prática de AF no parque $(p>0,05)$. Com exceção dos indicadores incidência de chuvas, poluição do ar e serviços de atendimento de emergência, todos os outros foram percebidos positivamente para prática de AF no parque.

Por meio do teste para diferença entre duas proporções, não foi verificada diferença na percepção positiva do parque urbano entre os usuários sedentários e ativos $(p>0,05)$. 
24 | EMC e percepção do ambiente para atividade física

Tabela 2. Comparação da percepção positiva dos indicadores ambientais do parque público por usuários sedentários e ativos

\begin{tabular}{|c|c|c|}
\hline \multirow{3}{*}{ Indicadores ambientais no parque } & \multicolumn{2}{|c|}{ Percepção positiva do parque urbano } \\
\hline & Sedentário & Ativo \\
\hline & $\%$ & $\%$ \\
\hline Incidência de chuvas & 13,2 & 11,7 \\
\hline Polvição do ar & 24,5 & 27,3 \\
\hline Beleza Geográfica & 100 & 98,2 \\
\hline Localização Geográfica & 92,5 & 97,0 \\
\hline Pista de caminhada/corrida & 90,6 & 95,8 \\
\hline Equipamentos disponíveis para $\mathrm{AF}$ & 79,2 & 72,7 \\
\hline Estacionamento no parque & 81,1 & 82,4 \\
\hline $\begin{array}{l}\text { Beleza arquitetônica das estruturas } \\
\text { construídas }\end{array}$ & 100 & 95,2 \\
\hline Programas públicos de AF & 69,8 & 57,6 \\
\hline Cartazes informativos sobre AF & 71,7 & 55,8 \\
\hline $\begin{array}{l}\text { Serviços de atendimento de } \\
\text { emergência }\end{array}$ & 54,7 & 47,9 \\
\hline Segurança pública nas imediações & 64,2 & 52,1 \\
\hline $\begin{array}{l}\text { Regulamentação de trânsito nas } \\
\text { Imediações }\end{array}$ & 79,2 & 69,7 \\
\hline Comportamento dos usuários & 84,9 & 87,9 \\
\hline Apoio e incentivos de amigos & 88,7 & 89,1 \\
\hline $\begin{array}{l}\text { Valor atribuído ao parque pela } \\
\text { Comunidade }\end{array}$ & 92,5 & 93,3 \\
\hline
\end{tabular}

\section{Discussão}

Ao analisar os EMC relacionados à $\mathrm{AF}$ dos usuários do parque, notou-se que a maioria está no estágio manutenção $(54,6 \%)$, o que indica serem ativos regularmente no mínimo há seis meses e, portanto, já adotaram uma conduta de AF no seu dia-a-dia (Marcus et al., 1992). Entretanto, incentivos devem ser dados para que esses usuários não abandonem este comportamento alcançado. Dos usuários investigados, 21,1\% encontrava-se no estágio ação, indicando que os mesmos são ativos regularmente a menos de seis meses. Marcus e Simkin (1994) comentam que os indivíduos que se encontram neste estágio têm grandes chances de recaída, pois a prática de AF regular ainda é recente, sendo necessário um grande esforço e tempo para que a adoção de uma conduta regular de AF seja 
efetivada. Desta forma, ambientes que não têm muitos indicadores positivos para prática de $A F$, sobretudo, os ligados ao suporte social, são prejudiciais às pessoas nesse estágio (Marcus \& Simkin, 1994; Marcus et al., 1992).

Em relação ao estágio preparação, 10,5\% dos usuários encontravam-se pronto para o início da prática regular de AF em um futuro próximo. No entanto, ainda não tinham assumido uma atitude efetiva. Neste estágio, os programas de intervenção de AF são fundamentais para solidificar a opção pela prática regular (Marcus \& Simkin, 1994). Desta maneira, programas permanentes de AF para a comunidade em parques urbanos são essenciais para que usuários que se encontram neste estágio sintam-se incentivados a adotar um estilo de vida ativo.

No estágio contemplação, foram encontrados $13,3 \%$ dos usuários. Embora os indivíduos neste estágio percebam o estilo de vida inativo como um problema e tenham a intenção de modificá-lo, ainda não se envolveram ou pensam que não estão prontos para uma tomada de ação efetiva (Marcus \& Simkin, 1994i Petroski \& Martins, 2008). Neste sentido, parques urbanos estruturados, com fácil acessibilidade e disponibilidade, infra-estrutura adequada, segurança e belezas naturais podem ajudar a derrubar barreiras pessoais para a AF.

Dos usuários analisados, somente $0,5 \%$ estava no estágio pré-contemplação. Contudo, atenção especial deve ser dada a estes sujeitos, pois eles têm como característica a suposição de que não precisam mudar o comportamento inadequado (Marcus \& Simkin, 1994). Pesquisadores comentam que ambientes com informações e campanhas sobre a importância da AF podem ajudar na quebra da resistência exercida pelos sujeitos deste estágio (Marcus \& Simkin, 1994; Norris et al., 2000; Petroski \& Martins, 2008). Assim, o parque urbano também tem que contar com iniciativas educacionais, ensinando e ressaltando os benefícios da prática da AF regular.

Ao agrupar as categorias do EMC, $24,3 \%$ dos usuários foram classificados com comportamento "sedentário" para a prática de AF e, 75,7\% com comportamento "ativo", situação esta desejada à saúde (Haskell, et al., 2007). Na literatura há relatos que frequentadores de parques urbanos já adotaram a $A F$ no seu cotidiano, pois a fazem de maneira regular neste ambiente (Bedimo-Rung et al., 2005; Kaczynski, Potwarka, \& Saelens, 2008). Porém, há outras pesquisas que relatam resultados contrários (Cohen, et al., 2007; Floyd, Spengler, Maddock, Gobster, \& Suau, 2008). Cohen et al. (2007) identificaram que a maioria dos usuários dos parques de Los Angeles é sedentária e a atividade mais comum é o piquenique. Floyd et al. (2008) verificaram que $70 \%$ dos frequentadores de parques públicos na 
Flórida fazem uso do ambiente para atividades sedentárias como o piquenique e a pescaria. No Brasil, um levantamento em parques públicos na zona oeste de São Paulo identificou que $73 \%$ dos frequentadores eram ativos no lazer (Oliveira, Bartholomeu, Tinucci, \& Forjaz, 2008). Tais achados evidenciam que ainda existem controvérsias na inter-relação AF e parques urbanos, o que necessita de mais investigações, sobretudo no Brasil.

Em relação às características sócio-demográficas, a maior parte dos usuários do Parque Jardim Botânico era do sexo masculino, de meia-idade, de NE médio a alto, com ensino superior completo e casado. Características semelhantes foram reportadas em usuários de outros parques urbanos (Cohen et al., 2007; Kaczynski et al., 2008).

A faixa etária foi associada significativamente com os EMC, indicando que a depender do grupo etário considerado o comportamento adotado em relação à AF sofre alteração. Na faixa etária inferior a 40 anos, houve uma maior proporção de usuários com comportamento "sedentário" (39\%). Já a maior proporção de usuários classificados nos EMC "ativo" foi nos adultos de meia-idade (87\%). Tais achados corroboram os resultados encontrados na literatura, que reporta serem as pessoas de meia-idade as que mais percebem positivamente o parque como ambiente agradável para AF e usufruem deste local para a prática de exercícios (Tinsley, Tinsley, \& Croskeys, 2002).

$O$ indicador incidência de chuvas em parques urbanos foi percebido negativamente para a prática de AF. Isto evidencia que fatores climáticos influenciam a percepção quanto à prática de exercícios, ademais, pesquisadores de outros países identificaram uma relação inversa entre incidência de chuvas e prática de AF (Chan, Ryan, \& Tudor-Locke, 2006). Infelizmente, a incidência de chuvas é um fator ambiental que não pode ser modificado, entretanto, a depender da estação do ano, no Brasil, a quantidade de precipitação de chuvas é diminuída e a utilização do parque para AF pode ser incentivada.

A prevalência de usuários com comportamento "ativo" (27,3\%) e "sedentário" $(24,5 \%)$ que perceberam o indicador poluição do ar no parque como positivo para $A F$, também foi baixa. Recentemente, a poluição do ar nas grandes cidades é considerada como uma das responsáveis pelos baixos níveis de AF das pessoas (Younger, Morrow-Almeida, Vindigni, \& Dannenberg, 2008). Tais evidências reforçam a necessidade de uma política de educação permanente contra a poluição atmosférica e conscientização em relação à preservação ambiental. 
Locais agradáveis, bastante arborizados, com ambientes construídos e naturais preservados são considerados propícios à prática de AF (Kruger, Carlson, \& Kohl, 2007; Mcginn, Evenson, Herring, Huston, \& Rodriguez, 2007), e isto ficou evidenciado no presente estudo, pois os indicadores beleza geográfica e beleza arquitetônica das estruturas construídas foram percebidos como indicadores estimuladores de AF em parques urbanos. Desta forma, a construção de parques urbanos deve levar em consideração um ambiente com belezas naturais (árvores e lagos) e construídas (jardins e monumentos).

A localização geográfica do parque foi percebida como um estímulo à prática de $A F$, independente do EMC. Pesquisadores internacionais (Cohen et al., 2007; Kaczynski et al., 2008) já enfatizaram que a construção de parques urbanos tem que ser próximo a áreas residenciais com acesso e disponibilidade para servir de facilitador às pessoas que moram na região. Assim, acessibilidade e disponibilidade são condições importantes a serem consideradas no planejamento urbano da construção destes ambientes de lazer.

Aspectos relacionados à infra-estrutura do ambiente no parque (pista de caminhada e corrida, equipamentos disponíveis para prática de $A F$, estacionamento no parque) e os aspectos políticos e campanhas sobre AF (programas públicos de AF) foram fatores percebidos positivamente para prática de exercícios independente dos EMC. Isto indica que a implantação de ambientes propícios de infra-estrutura com materiais disponíveis, como barras, bancos, espaldares, programas de educação e realização de AF no parque podem aumentar os níveis de AF das pessoas, como já foi reportado em outros países (Bedimo-Rung et al., 2005; Kruger et al., 2007).

Para o indicador de serviços de atendimentos de emergência no parque, os usuários, com comportamento sedentário, perceberam mais positivamente este aspecto. Tais achados não dão uma segurança de afirmar que este indicador é percebido como positivo para prática de AF em parques. Ademais, não foi encontrada na literatura pesquisada, estudos que fizeram a relação deste aspecto com a AF, o que dificulta maiores inferências sobre o assunto.

Outro indicador percebido como um estímulo à prática de AF foi o de segurança pública nas imediações do parque. Tais resultados indicam que a falta de segurança no ambiente de lazer e no contexto que cerca o local, como nas ruas próximas e no bairro, são aspectos que impedem as pessoas de se exercitarem. Pesquisadores internacionais encontraram uma associação positiva entre a alta criminalidade, assaltos e violência com os baixos níveis de AF das pessoas (Cohen et al., 2007). 
Desta maneira, há uma necessidade da presença dos setores de segurança pública no planejamento da construção de ambientes de lazer, para que medidas de combate e controle da violência e crime sejam permanentemente tomadas.

A regulamentação do trânsito nas imediações do parque também foi um indicador percebido positivamente para prática de AF independente dos EMC. Em geral, a regulamentação do trânsito em qualquer ambiente é reportada como um facilitador para a AF (Mcginn et al., 2007; Tinsley et al., 2002). Assim, entende-se que a regulamentação do trânsito com ruas bem sinalizadas e o tráfego controlado, podem servir para que um ambiente de lazer seja frequentado. Isto alerta sobre a importância que outros setores públicos têm no planejamento de parques urbanos e na ajuda para mudança de comportamento das pessoas.

Outros indicadores ambientais percebidos no presente estudo como positivos para a prática de $\mathrm{AF}$ foram os de valores e atitudes, como o de comportamento positivo dos usuários do parque em relação à prática de $A F$, apoio e incentivos de amigos para praticar exercícios e o valor atribuído ao parque pela comunidade. Todos os três indicadores analisados são reportados na literatura como fundamentais no incentivo para o aumento dos níveis de AF das pessoas (Kirchhoff, Elliott, Schlichting, \& Chin, 2008).

Os pontos positivos da presente investigação que merecem destaque são: a) a verificação dos $\mathrm{EMC}$ relacionados à $\mathrm{AF}$ em usuários de parques urbanos, pois não foram encontrados na literatura pesquisada estudos que fizeram esta análise, b) ter sido um dos poucos no país a identificar indicadores percebidos como positivos para prática de $\mathrm{AF}$ em parques urbanos, c) a investigação dos indicadores ambientais tanto em pessoas que já adotaram a prática regular de AF quanto em pessoas que não adotaram, o que oferece uma visão geral para políticas públicas de promoção de AF em ambientes de lazer, d) o uso de um instrumento de percepção do ambiente criado e validado no Brasil.

O presente estudo foi o primeiro a identificar a percepção dos usuários ativos e sedentários acerca dos indicadores ambientais de parques urbanos do Brasil, sugerindo, desta forma, que ambientes públicos de lazer devem levar em consideração o suporte social e ser atrativo para toda a família, visando uma maior integração entre a comunidade e o local. Entretanto, algumas limitações fazem-se necessárias destacar: a investigação de usuários de um único parque urbano, o que impossibilita a extrapolação dos dados para outras localidades do país. Portanto, fazem-se necessárias outras pesquisas nos diversos parques urbanos do Brasil para comparar os dados encontrados, tendo em vistas uma futura verificação da 
efetividade de políticas públicas de AF voltada à comunidade. Outra limitação do estudo está relacionada à seleção da amostra que contou somente com as pessoas que devolveram os instrumentos via correio, impossibilitando saber se as pessoas que não enviaram $o$ instrumento percebem da mesma maneira o ambiente existente. Além destas, a falta da análise de cada EMC em relação à percepção dos indicadores ambientais do parque, pois impossibilita o conhecimento da percepção do ambiente para cada comportamento observado.

Uma outra limitação da presente pesquisa está relacionada com a validade interna do constructo do questionário, embora a escala tenha apresentado uma matriz teórica bem estabelecida e confiável (Reis et al., 2002). Entretanto, o estudo adiciona informações importantes sobre usuários de parques urbanos, podendo servir como parâmetro comparativo para investigações em diferentes localidades. De acordo com o que foi observado, pode-se concluir que a maior parte dos usuários investigados é ativo regularmente há mais de seis meses, sobretudo, os indivíduos de meia-idade. Com exceção dos indicadores incidência de chuvas, poluição do ar e serviços de atendimento de emergência, todos os outros foram fortemente percebidos como positivos para prática de AF em parques, sendo que esta percepção não diferiu entre usuários com comportamento "sedentário" e "ativo".

Por fim, recomenda-se que iniciativas de promoção da AF sejam tomadas no parque investigado com a finalidade de estimular a adoção de prática regular de AF entre os sujeitos com comportamento sedentário e a manutenção entre os indivíduos ativos regularmente.

\section{Bibliografia}

Associação Brasileira de Empresas de Pesquisa - Critério de Classificação Econômica Brasil (2003). Consultado on-line a partir de: http://www.abep.org/codigosguias/ ABEP_CCEB.pdf

Bedimo-Rung, A. L., Mowen, A. J. \& Cohen, D. A. (2005). The significance of parks to physical activity and public health: A conceptual model. American Journal of Preventive Medicine, 28, 159-168.

Cervantes, O., Espejel, I., Arellano, E. \& Delhumeau, S. (2008). Users' perception as a tool to improve urban beach planning and management. Environmental Management, 42, 249-264.

Chan, C. B., Ryan, D. A. \& Tudor-Locke, C. (2006). Relationship between objective measures of physical activity and weather: A longitudinal study. The International Journal of Behavioral Nutrition and Physical Activity, 3. Consultado on-line a partir de http://www.ijbnpa.org/content/3/1/21. doi: 10.1186/1479-5868-3-21 
Cohen, D. A., McKenzie, T. L., Sehgal, A., Williamson, S., Golinelli, D. \& Lurie, N. (2007). Contribution of public parks to physical activity. American Journal of Public Health, 97, 509-514.

Cohen, S. J., Meister, J. S. \& Zapien, J. G. (2004). Special action groups for policy change and infrastructure support to foster healthier communities on the Arizona-Mexico border. Public Health Reports, 119, 40-47.

Cunningham, C. O., Shapiro, S., Berg, K. M., Sacajiu, G., Paccione, G. \& Goulet, J. L. (2005). An evaluation of a medical outreach program targeting unstably housed HIV-infected individuals. Journal of Health Care for the Poor and Underserved, 16, 127-138.

Floyd, M. F., Spengler, J. O., Maddock, J. E., Gobster, P. H. \& Suau, L. J. (2008). Park-based physical activity in diverse communities of two U.S. cities. An observational study. American Journal of Preventive Medicine, 34, 299-305.

Green, L.W., Glanz, K., Hochbaum, G.M., Kok, G., Kreuter, M.W., Lewis, F.M., et al. (1994). Can we build on, or must we replace, the theories and models in health education? Health Education Research, 9(3), 397-404.

Haskell, W. L., Lee, I. M., Pate, R. R., Powell, K. E., Blair, S. N., Franklin, B. A., et al. (2007). Physical activity and public health: Updated recommendation for adults from the American College of Sports Medicine and the American Heart Association. Circulation, 116, 1081-1093.

Kaczynski, A. T., Potwarka, L. R. \& Saelens, B. E. (2008). Association of park size, distance, and features with physical activity in neighborhood parks. American Journal of Public Health, 98, 1451-1456.

Kirchhoff, A. C., Elliott, L., Schlichting, J. A. \& Chin, M. H. (2008). Strategies for physical activity maintenance in African-American women. American Journal of Health Behavior, 32, 517-524.

Kruger, J., Carlson, S. A. \& Kohl, H. W. (2007). Fitness facilities for adults: differences in perceived access and usage. American Journal of Preventive Medicine, 32, 500-505.

Librett, J., Henderson, K., Godbey, G. \& Morrow JR J. R. (2007). An introduction to parks, recreation, and public health: Collaborative frameworks for promoting physical activity. Journal of Physical Activity \& Health, 4, S1-S13.

Marchisio, S., Sarasino, D., Manzoli, L., Di Stanislao, F. \& Panella, M. (2007). Evaluation of the work environment in a local healthcare organisation in Italy: Practical aspects and Public Health relevance. Igiene e Sanità Pubblica, 63, 361-375.

Marcus, B. H. \& Simkin, L. R. (1994). The trans-theoretical model: Applications to exercise. Medicine and Science in Sports and Exercise, 26, 1400-1404.

Marcus, B. H., Rossi, J. S., Selby, V. C., Niaura, R. S. \& Abrams, D. B. (1992). The stages and processes of exercise adoption and maintenance in a worksite sample. Health Psychology, 11(6), 386-395.

Mcginn, A. P., Evenson, K. R., Herring, A. H., Huston, S. L. \& Rodriguez, D. A. (2007). Exploring associations between physical activity and perceived and objective measures of the built environment. Journal of Urban Health, 84, 162-184. 
Mitra, A. \& Lankford, S. (1999). Research methods in park, recreation and leisure services. Champaign, IL: Sagamore Publishing.

Nigg, C. R. (2005). There is more to stages of exercise than just exercise. Exercise and Sport Sciences Reviews, 33, 32-35.

Norris, S. L., Grothaus, L. C., Buchner, D. M. \& Pratt, M. (2000). Effectiveness of physician based assessment and counseling for exercise in a staff model HMO. Preventive Medicine, 30, 513-523.

Oliveira, G. F., Bartholomeu, T., Tinucci, T. \& Forjaz, C. L. M. (2008). Risco cardiovascular de usuários ativos, insuficientemente ativos e inativos de parques públicos. Revista Brasileira de Cineantropometria e Desempenho Humano, 10, 170-175.

Petroski, E. L. \& Martins, M. O. (2008). Atividade física de lazer e estágios de mudança de comportamento em professores universitários. Revista Portuguesa de Ciências do Desporto, 8, 209-218.

Prochaska, J., DiClemente, C. (1982). Transtheoretical therapy: Toward a more integrative model of change. Psychotherapy: Theory, Research and Practice, 19(3), 276-288.

Reis, R. S., Nascimento, J. V. \& Petroski, E. L. (2002). Escala de autopercepção do ambiente para a realização de atividades físicas. Revista Brasileira de Atividade Física e Saúde, 7, 14-23.

Righi, E., Aggazzotti, G., Fantuzzi, G., Ciccarese, V. \& Predieri, G. (2002). Air quality and well-being perception in subjects attending university libraries in Modena (Italy). The Science of the Total Environment, 286, 41-50.

Tinsley, H. E., Tinsley, D. J. \& Croskeys, C. E. (2002). Park usage, social milieu, and psychosocial benefits of park use reported by older urban park users from four ethnic groups. Leisure Sciences, 24, 199-218.

Younger, M., Morrow-Almeida, H. R., Vindigni, S. M. \& Dannenberg, A. L. (2008). The built environment, climate change, and health: Opportunities for co-benefits. American Journal of Preventive Medicine, 35, 517-526. 